\title{
Overexpression of endothelial nitric oxide synthase accelerates atherosclerotic lesion formation in apoE-deficient mice
}

\author{
Masanori Ozaki, ${ }^{1}$ Seinosuke Kawashima,,${ }^{1}$ Tomoya Yamashita, ${ }^{1}$ Tetsuaki Hirase,${ }^{1}$ \\ Masayuki Namiki, ${ }^{1}$ Nobutaka Inoue,${ }^{1}$ Ken-ichi Hirata, ${ }^{1}$ Hiroyuki Yasui, ${ }^{2}$ \\ Hiromu Sakurai, ${ }^{2}$ Yuichi Yoshida, ${ }^{3}$ Masahiro Masada, ${ }^{3}$ and Mitsuhiro Yokoyama ${ }^{1}$ \\ ${ }^{1}$ Division of Cardiovascular and Respiratory Medicine, Department of Internal Medicine, \\ Kobe University Graduate School of Medicine, Kobe, Japan \\ ${ }^{2}$ Department of Analytical and Bioinorganic Chemistry, Kyoto Pharmaceutical University, Kyoto, Japan \\ ${ }^{3}$ Laboratory of Biochemistry, Faculty of Horticulture, Chiba University, Chiba, Japan
}

\begin{abstract}
Nitric oxide (NO) derived from endothelial NO synthase (eNOS) is regarded as a protective factor against atherosclerosis. Therefore, augmentation of eNOS expression or NO production by pharmacological intervention is postulated to inhibit atherosclerosis. We crossed eNOS-overexpressing (eNOS-Tg) mice with atherogenic apoE-deficient (apoE-KO) mice to determine whether eNOS overexpression in the endothelium could inhibit the development of atherosclerosis. After 8 weeks on a high-cholesterol diet, the atherosclerotic lesion areas in the aortic sinus were unexpectedly increased by more than twofold in apoE-KO/eNOS-Tg mice compared with apoE-KO mice. Also, aortic tree lesion areas were approximately $50 \%$ larger in apoE-KO/eNOS-Tg mice after 12 weeks on a high-cholesterol diet. Expression of eNOS and NO production in aortas from apoE-KO/eNOS-Tg mice were significantly higher than those in apoE-KO mice. However, eNOS dysfunction, demonstrated by lower NO production relative to eNOS expression and enhanced superoxide production in the endothelium, was observed in apoE-KO/eNOS-Tg mice. Supplementation with tetrahydrobiopterin, an NOS cofactor, reduced the atherosclerotic lesion size in apoE-KO/eNOS-Tg mice to the level comparable to apoE-KO mice, possibly through the improvement of eNOS dysfunction. These data demonstrate that chronic overexpression of eNOS does not inhibit, but accelerates, atherosclerosis under hypercholesterolemia and that eNOS dysfunction appears to play important roles in the progression of atherosclerosis in apoE-KO/eNOS-Tg mice.
\end{abstract}

J. Clin. Invest. 110:331-340 (2002). doi:10.1172/JCI200215215.

\section{Introduction}

Nitric oxide (NO), constitutively produced by endothelial nitric oxide synthase (eNOS), is a potent powerful vasodilator and possesses various vasoprotective effects such as inhibition of platelet aggregation, suppression of adhesion of leukocytes or monocytes on the endothelial surfaces, and inhibition of proliferation and migration of vascular smooth muscle cells. Because of these biological actions, eNOS-derived NO is regarded as an antiatherogenic factor. The observation of

Received for publication February 6, 2002, and accepted in revised form June 3, 2002.

Address correspondence to: Seinosuke Kawashima, Division of Cardiovascular and Respiratory Medicine, Department of Internal Medicine, Kobe University Graduate School of Medicine, 7-5-1, Kusunoki-cho, Chuo-ku, Kobe, 650-0017, Japan. Phone: 81-78-382-5842; Fax: 81-78-382-5859;

E-mail: kawashim@med.kobe-u.ac.jp.

Conflict of interest: No conflict of interest has been declared. Nonstandard abbreviations used: nitric oxide (NO); endothelial nitric oxide synthase (eNOS); eNOS transgenic (eNOS-Tg); apoE-deficient (apoE-KO); tetrahydrobiopterin $\left(\mathrm{BH}_{4}\right)$; diaminofluorescein-2 diacetate (DAF-2 DA); 2-methyl-6(4-methoxyphenyl)-3, 7-dihydroimidazol [1, 2-a] pyrazin-3-onehydrochloride (MCLA); wild-type (WT); inducible NOS (iNOS). impaired endothelium-dependent vasorelaxation in hypercholesterolemia indicates that the loss of NO bioactivity is an early feature of atherosclerosis $(1,2)$. Therefore, preservation of NO bioactivity in the vessel walls might be one of the therapeutic strategies to inhibit the development of atherosclerosis. Indeed, administration of NO precursor L-arginine is demonstrated to restore the depressed endothelium-dependent vasodilatation in hypercholesterolemic rabbits and humans $(3,4)$. In addition, chronic administration of L-arginine is exhibited to cause the regression of preexisting intimal lesions through the restoration of $\mathrm{NO}$ bioactivity (5) and to inhibit the progression of atherosclerosis in cholesterol-fed rabbits and LDL receptor-deficient mice $(6,7)$. Therefore, upregulation of eNOS expression or restored NO bioactivity has been expected to inhibit the development of atherosclerosis.

Recently, the gene therapy of NOS was designed to increase or restore vascular $\mathrm{NO}$ production under the pathological situations in which the bioactivity of $\mathrm{NO}$ is reduced. In vivo gene transfer of eNOS or neuronal NOS is demonstrated to improve or augment NOmediated vasorelaxation in atherosclerotic arteries (8). Furthermore, the gene transfer of eNOS could reduce 
adhesion molecule expression and inflammatory cell infiltration in carotid arteries of cholesterol-fed rabbits (9). Accordingly, the gene transfer of NOS has been expected as a favorable candidate of therapy for the prevention or inhibition of atherosclerosis. However, these beneficial effects are examined only in the short term by using a pharmacological approach or transient gene transfer. Furthermore, NOS itself produces superoxide anions in the absence of its substrate L-arginine or cofactors $(10,11)$. The long-term outcomes of gene delivery of NOS remain unclear, and its effects on vascular structures are undetermined. From these viewpoints, the actual roles of eNOS in the development of atherosclerosis should be examined by using the model that chronically overexpresses eNOS at the vessel walls.

In this study, we aimed to investigate the long-term effects of eNOS overexpression in the endothelium on the development of atherosclerosis by using genetically engineered mice. eNOS transgenic (eNOS-Tg) mice, which chronically overexpress eNOS in the vascular endothelium, show various vasoprotective effects on different vascular injury models by overproducing endothelium-derived NO (12-14). ApoE-deficient (apoE-KO) mice, a well-known animal model of atherosclerosis whose lesion development is similar to that observed in humans (15), were crossed with eNOS-Tg mice to generate eNOS-overexpressing apoE-KO (apoE-KO/eNOS-Tg) mice. We found promotion of atherosclerosis in apoE-KO/eNOS-Tg mice in comparison with apoE-KO mice, possibly due to the dysfunction of overexpressed eNOS in the endothelium that resulted in decreased NO bioactivity and enhanced production of superoxide. Supplementation with a NOS cofactor tetrahydrobiopterin $\left(\mathrm{BH}_{4}\right)$ reversed eNOS dysfunction and inhibited atherosclerotic progression. Our study provides evidence that the preservation of eNOS function rather than eNOS expression is essential for the prevention of atherosclerosis.

\section{Methods}

Materials. Diaminofluorescein-2 diacetate (DAF-2 DA) and 2-methyl-6-(4-methoxyphenyl)-3, 7-dihydroimidazol [1, 2-a] pyrazin-3-one-hydrochloride (MCLA) were purchased from Daiichi Pure Chemicals Co. (Tokyo, Japan) and Tokyo Kasei Kogyo Co. (Tokyo, Japan), respectively. Sapropterin hydrochloride, chemically synthesized $\mathrm{BH}_{4}$, was obtained from Suntory Ltd. (Kyoto, Japan). The following Ab's were used: a rabbit polyclonal anti-eNOS Ab (Transduction Laboratories, Lexington, Kentucky, USA); a rat monoclonal anti-mouse monocyte/macrophage $\mathrm{Ab}$ (MOMA-2; Biosource International, Camarillo, California, USA); a monoclonal anti- $\alpha$ smooth muscle actin Ab (DAKO A/S, Glostrup, Denmark). All other chemicals were obtained from Sigma-Aldrich (St. Louis, Missouri, USA).

Animal preparation. Homozygous apoE-KO mice (50\% C57BL/6J, 50\% 129SVJ) (16) were backcrossed at least four times to C57BL/6J mice $(93.8 \%$ C57BL/6J, 7.2\% 129SVJ). eNOS-Tg mice (99.9\% C57BL/6J) overexpress- ing bovine eNOS under the control of preproendothelin-1 promoter have been described previously (12). Heterozygous eNOS-Tg mice were crossed with apoE-KO mice to yield double-heterozygous mice $(96.9 \%$ C57BL/6J, 3.1\% 129SVJ). These heterozygous littermates were bred with apoE-KO mice to establish apoE-KO/eNOS-Tg mice. The animals used in this experiment were offspring of an intercross between apoE-KO/eNOS-Tg mice and apoE-KO mice $(96.9 \%$ C57BL/6J, 3.1\% 129SVJ). PCR was performed for genotyping for apoE (16) and the eNOS transgene (12).

Experimental design. ApoE-KO mice $(n=86)$ and apoE-KO/eNOS-Tg mice $(n=92)$ were weaned at 4 weeks of age onto a high-cholesterol diet $(1.25 \%$ cholesterol, $7.5 \%$ cocoa butter, $7.5 \%$ casein, $0.5 \%$ sodium cholate; Oriental Yeast Co., Tokyo, Japan) (17) and maintained the diet for 8 or 12 weeks. In separate experiments, apoE-KO mice $(n=21)$ and apoE$\mathrm{KO} / \mathrm{eNOS}-\mathrm{Tg}$ mice $(n=36)$ were maintained on a highcholesterol diet supplemented with $10 \mathrm{mg} / \mathrm{kg} /$ day $\mathrm{BH}_{4}$ as described previously (18). eNOS-Tg mice $(99.9 \%$ $\mathrm{C} 57 \mathrm{BL} / 6 \mathrm{~J})$ were used in the experiments of immunoblotting for eNOS expression $(n=6)$ and NOS activity $(n=6)$. Their littermate wild-type (WT) mice were used for a control group in the experiments of immunoblotting for eNOS expression $(n=6)$, NOS activity $(n=6)$, and in situ superoxide detection $(n=14)$. Animals were provided the diet and water ad libitum and were maintained on a 12-hour light/dark cycle. All animal experiments were conducted according to the Guidelines for Animal Experiments at Kobe University Graduate School of Medicine.

Histological analysis of atherosclerotic lesions. The apoE-KO and apoE-KO/eNOS-Tg mice were euthanized at the age of 12 or 16 weeks, respectively ( 8 or 12 weeks on the high-cholesterol diet, respectively), and the atherosclerotic lesions were analyzed as described previously (19, 20). After mice were anesthetized with pentobarbital sodium $(80 \mathrm{mg} / \mathrm{kg}$ intraperitoneally; Abbot Laboratories, Abbott Park, Illinois, USA), the aorta was perfused with normal saline containing $10 \mathrm{U} / \mathrm{ml}$ heparin. Then the aortic sample was dissected from the middle of left ventricle to the iliac bifurcation and fixed in $4 \%$ paraformaldehyde overnight. The samples were cut in the ascending aorta, and the proximal samples containing the aortic sinus were embedded in OCT compounds (Tissue-Tek; Sakura Finetechnical Co., Tokyo, Japan) and sectioned (10- $\mu \mathrm{m}$ thickness). Five consecutive sections, spanning $550 \mu \mathrm{m}$ of the aortic root, were collected from each mouse and stained with Sudan III and Masson's trichrome. For quantitative analysis of the atherosclerosis, the total lesion area of five sections from each mouse was measured with the NIH 1.61 Imaging Software by modifying the method reported previously (17). The distal part of the excised aorta (from aortic arch to iliac bifurcation) was dissected free from surrounding tissues, opened longitudinally, and pinned onto a silicon-coated dish. Image analysis was performed on Sudan III-stained aortas with the NIH 
1.61 Imaging Software. The amount of aortic lesion formation in each animal was measured as the percentage of lesion area per total area of the aorta (20).

Immunohistochemistry. Immunohistochemical staining for MOMA- 2 and $\alpha$-smooth muscle actin in atherosclerotic lesions in the aortic root were performed by the labeled streptavidin biotin method as described previously $(12,13)$. Quantitative analysis was shown as a percentage of the positive-stained area in the total atherosclerotic lesion area as described previously (21).

Hemodynamic analysis. Systemic blood pressure and heart rate were measured in WT, eNOS-Tg, apoE-KO, and apoE-KO/eNOS-Tg mice at 16 weeks of age under the conscious and unrestrained conditions as described previously (12). Blood pressure and heart rate were continuously measured and recorded on a Macintosh computer with MacLab system (Bioresearch Center, Nagoya, Japan) at least 4 hours after recovery from anesthesia.

Plasma lipid analysis. After mice were deprived of food for at least 12 hours, blood was collected by the cardiac puncture into heparin-coated tubes. Plasma was obtained through centrifugation of the blood for 15 minutes at $5,500 \mathrm{~g}$ at $4{ }^{\circ} \mathrm{C}$ and stored at $-80^{\circ} \mathrm{C}$ until each assay was performed. Concentration of total cholesterol and triglyceride were determined using an automated clinical chemistry analyzer. HDL-cholesterol levels were quantified by enzymatic reaction using a commercially available kit (Wako Pure Chemicals Industries, Osaka, Japan).

Protein analysis for eNOS. The protein samples were extracted from the mice at the age of 12 weeks. The expression of eNOS in aortas was analyzed by immunoblotting, and $\mathrm{Ca}^{2+}$-dependent NOS enzymatic activity was determined by conversion of $\left[{ }^{3} \mathrm{H}\right]$-L-arginine to $\left[{ }^{3} \mathrm{H}\right]$-L-citrulline as described previously (12). Enzyme activity was expressed as citrulline production in picomoles per milligram of protein per minute.

In situ fluorescent signal detection and quantitative measurements of NO in aortas. NO production from aortas in situ was detected with fluorescence indicator DAF-2 DA (22-24). The fluorescent images of NO were obtained with a luminograph (LB981, NightOWL imaging system; EG\&G Berthold, Bad Wildbad, Germany) as described previously (25). Briefly, the aorta containing aortic arch to iliac bifurcation was excised from 12week-old animals, and adventitial tissues were dissected promptly in PBS ( $\mathrm{pH}$ 7.4). The specimen was pinned onto a black-colored dish filled with $0.1 \mathrm{~mol} / 1$ phosphate buffer ( $\mathrm{pH} 7.4$ ) containing $1.5 \mathrm{mmol} / 1$ calcium chloride and placed in a light-tight box to prevent interference by external light. DAF-2 DA was then added into the solution to yield the final concentration of 10 $\mu \mathrm{mol} / 1$. After 5 minutes of incubation, the sample was excited by blue light through the filter (485 nm). The emitted fluorescent image caused by the reaction of DAF-2 DA with NO was obtained by the luminograph with a band-pass filter (center of $532 \mathrm{~nm}$ ), and its signal intensity was measured at the wavelength of $515 \mathrm{~nm}$ for 1 second. The aortic tissue was subsequently incubated in the presence of $1 \mu \mathrm{mol} / 1$ acetylcholine for 5 minutes, and the fluorescent signal image was again measured. After each experiment, the examined samples were stained with Sudan III to discriminate the atherosclerotic plaque from nonplaque areas.

The output data of fluorescent signal intensity due to $\mathrm{NO}$ was recorded and quantitatively analyzed by WinLight 32 software (the NightOWL-imaging system) for nonplaque areas of aorta. To exclude the interference of nonspecific emission and reflection from aortic collagen fibers, the background image was subtracted from each of the light-emission images after DAF-2 DA reaction. At least ten areas in the aorta (0.0005-0.001 $\left.\mathrm{cm}^{2}\right)$ were randomly selected, and the fluorescent signals were measured in every area. The amounts of NO production were expressed as picowatts per centimeter square. NO production from the endothelium was estimated by subtracting the fluorescent signal under the basal condition from that under the acetylcholine-stimulated condition.

In situ detection and quantitative measurements of superoxide in aortas. Spatial distribution and quantitative determination of superoxide production was examined by chemiluminescent detection with a sensitive and specific probe, MCLA, which is a Cypiridina luciferin analogue (25-27). The chemiluminescent images were obtained by the NightOWL luminograph at the wavelength of $465 \mathrm{~nm}$ (25). Briefly, aortas excised from 12week-old animals were dissected free from the surrounding tissues and mounted in a black-colored silicon dish filled with PBS at pH 7.4. After the samples were placed in a light-tight box to prevent interference by external light, MCLA was applied to the chamber (final concentration: $20 \mu \mathrm{mol} / \mathrm{l}$ ). The light emission due to the in situ MCLA reaction with superoxide anion was measured for 5 minutes, and the measurement was repeated three times to confirm the reproducibility. The last signal image was used for the quantitative analysis. Subsequently, the endothelium was removed, and the chemiluminescent signal image was repeatedly obtained. The chemiluminescence due to superoxide was identified by application of $\mathrm{Cu} / \mathrm{Zn}$ SOD $(400 \mathrm{U} / \mathrm{ml})$ at the end of each measurement. The examined samples were then stained with Sudan III.

The output data of light emission was recorded as images of a nonlinear gray scale that were converted to the pseudocolor. The chemiluminescence signal intensities due to superoxide production were quantitatively analyzed with WinLight 32 software. Quantification of superoxide production was evaluated separately in approximately ten randomly selected sites $\left(0.0012-0.002 \mathrm{~cm}^{2}\right)$ of either plaque or nonplaque area in aortic vessels that were discriminated by Sudan III staining. Measurements from each area were averaged in plaque or nonplaque area per animal after subtraction of the background signal. Quantification of superoxide production was expressed as the ratio (fold increase) to the average values obtained from WT mice. The reduced superoxide levels by endothelial 


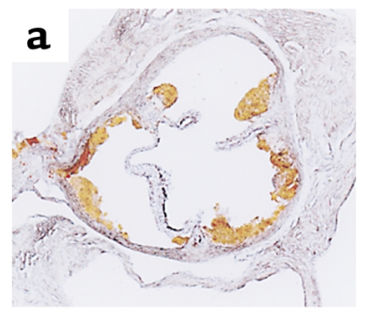

ApoE-KO

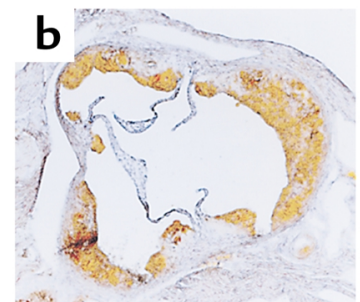

ApoE-KO/eNOS-Tg c

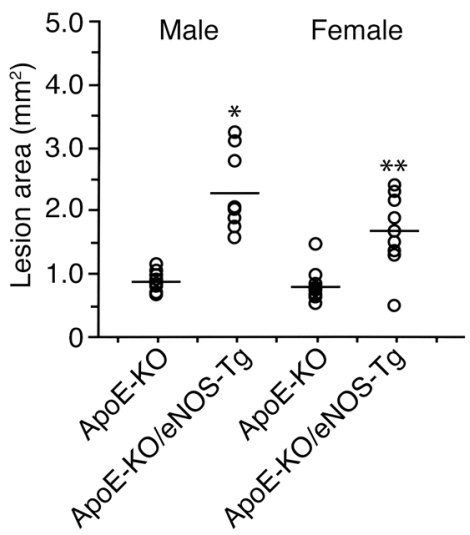

removal were expressed as a percentage of the reduced signal intensities to those before denudation.

$\mathrm{BH}_{4}$ contents in aortas. Biopterin contents in aortas were measured by HPLC analysis as described previously $(18,28)$. The amount of $\mathrm{BH}_{4}$ was calculated from the difference between the total $\left(\mathrm{BH}_{4}\right.$ plus $\mathrm{BH}_{2}$ plus oxidized biopterin) and alkaline-stable biopterin $\left(\mathrm{BH}_{2}\right.$ plus oxidized biopterin).

Statistical analysis. Data were expressed as mean plus or minus SD. An unpaired Student $t$ test was used to detect significant differences when two groups were compared. One-way ANOVA was used to compare the differences among three or four groups, with Bonferroni's test for post hoc analysis. $P$ values less than 0.05 were considered statistically significant.

\section{Results}

Accelerated atherosclerosis by eNOS overexpression in apoE-KO mice. After 8 weeks on a high-cholesterol diet (at the age of 12 weeks), atherosclerotic lesion formation was observed in the aortic sinus of apoE-KO mice (Figure 1a). In apoE-KO/eNOS-Tg mice, unexpectedly, the atherosclerotic lesions were markedly promoted compared with apoE-KO mice (Figure 1b). As shown in Figure 1c, the lesion size was significantly increased 2.6-fold in male and twofold in female apoE-KO/eNOS-Tg mice compared with apoE-KO mice. In histological examination, the atherosclerotic plaque areas were remarkably greater in apoE-KO/eNOS-Tg mice than in apoE-KO mice (Figure 2). The percentage of MOMA-2-stained area in the plaque was not significantly different between the two groups (apoE-KO mice: $64 \% \pm 14 \%$; apoE$\mathrm{KO} / \mathrm{eNOS}$-Tg: $61 \% \pm 12 \%, P=\mathrm{NS})$. The $\alpha$-smooth muscle actin-positive cells were few or hardly detectable (data not shown), and fibrotic changes were only partly dis-

\section{Figure 1}

Atherosclerotic lesions in the aortic sinus. (a and $\mathbf{b}$ ) Representative photographs of Sudan III-stained aortic root sections from apoE-KO and apoE-KO/eNOS-Tg mice fed on a high-cholesterol diet for 8 weeks. Sections were taken at the same level of aortic valves (original magnification $\times 15$ ). (c) Quantitative analysis of atherosclerotic lesion size in males and females of apoE-KO and apoE-KO/eNOS-Tg mice. Total lesion area of five sections in the aortic root from each mouse was quantified morphometrically as described in Methods. Each symbol represents the lesion area measurement from an individual mouse, with the mean per group indicated by a horizontal line. After 8 weeks on a high-cholesterol diet, the atherosclerotic lesion areas were significantly increased in apoE-KO/eNOS-Tg compared with apoE-KO mice. ${ }^{*} P<0.001$ vs. male apoE-KO mice; ${ }^{*} P<0.01$ vs. female apoE-KO mice.

tributed in the plaque lesions of both apoE-KO and apoE-KO/eNOS-Tg mice (Figure 2, c and d). Thus, the atherosclerotic lesions were more extended in apoE$\mathrm{KO} / \mathrm{eNOS}-\mathrm{Tg}$ mice, whereas the cellular composition was not changed between the two groups.

The mean lesion areas in the aortic tree statistically did not differ between apoE-KO and apoE-KO/eNOS$\mathrm{Tg}$ mice after 8 weeks on a high-cholesterol diet (percentage of lesion area: $8.8 \% \pm 3.0 \%$ in male apoE-KO mice; $10.4 \% \pm 2.4 \%$ in male apoE-KO/eNOS-Tg; $8.3 \% \pm 2.8 \%$ in female apoE-KO mice; $10.8 \% \pm 2.3 \%$ in female apoE-KO/eNOS-Tg; $P=\mathrm{NS}$ ). For this reason, we extended the cholesterol feeding period for an additional 4 weeks. After 12 weeks on a high-cholesterol diet, the lesion formation in the aortic tree was also markedly progressed in apoE-KO/eNOS-Tg mice compared with apoE-KO mice (Figure 3, a and b).
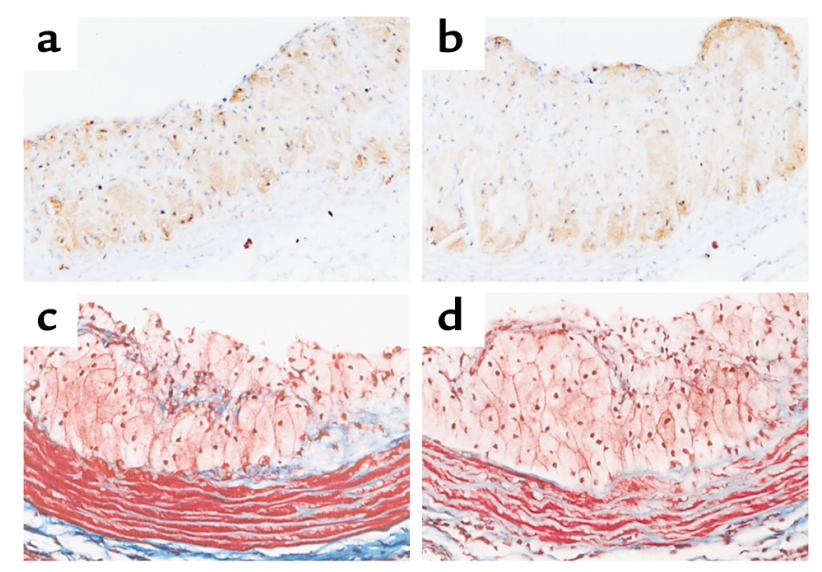

\section{Figure 2}

Histological examination of atherosclerotic lesions in the aortic root. ( $\mathbf{a}$ and $\mathbf{b}$ ) Photographs are representative of immunostaining for MOMA-2 in the atherosclerotic lesions, which showed no difference in the distribution between apoE-KO (a) and apoE-KO/eNOS-Tg mice (b) fed on a high-cholesterol diet for 8 weeks. (c and d) Fibrotic changes were detected with Masson's trichrome in the aortic root sections from apoE-KO (c) and apoE-KO/eNOS-Tg mice (d) fed a high-cholesterol diet. Fibrosis was only partly distributed in the plaque lesions of both mice (original magnification $\times 75$ ). 

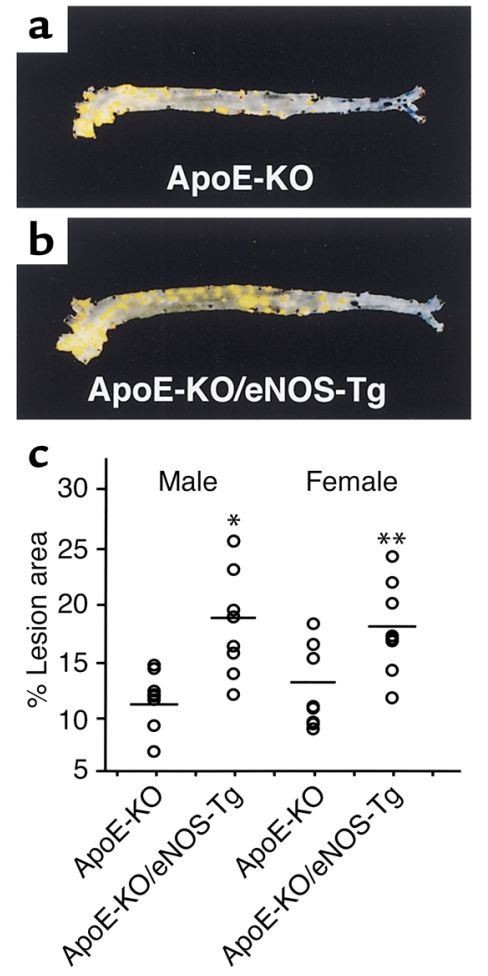

The quantitative analysis showed significant increases in the lesion area of 1.5 times in males and 1.4 times in females compared with apoE-KO mice (Figure 3c). Thus, atherosclerotic development was significantly accelerated by eNOS overexpression in the endotheli$\mathrm{um}$ in the early stage of atherosclerosis.

Plasma lipids levels and hemodynamics. Plasma cholesterol levels were remarkably elevated by a high-cholesterol feeding in both apoE-KO and apoE-KO/eNOS-Tg mice; however, there was no significant difference between the two groups (Table 1). In contrast, triglyceride levels were not affected by a high-cholesterol diet in both groups (Table 1). Mean systemic blood pressure in apoE-KO/eNOS-Tg mice was $83.2 \pm 7.4 \mathrm{mmHg}(n=8)$, which was approximately $20 \mathrm{mmHg}$ lower than that in apoE-KO mice $(103.5 \pm 5.6 \mathrm{mmHg} ; n=7, P<0.01)$. As reported previously (12), eNOS overexpression in the endothelium significantly decreased blood pressure in apoE-KO mice.

Expression of eNOS and NOS activity in aortas. As shown in Figure 4, a and b, eNOS expression in aortas from eNOS-Tg mice was significantly greater than in WT

\section{Figure 3}

Atherosclerotic lesions in aortas. (a and b) Sudan III-stained, longitudinally opened aortas from apoE-KO (a) and apoE-KO/eNOS-Tg mice (b) at the age of 16 weeks. (c) Quantitative analysis of atherosclerotic lesion size in 16-week-old mice. After 12 weeks on a high-cholesterol diet, the lesion size in apoE-KO/eNOS-Tg mice was significantly greater than that in apoE-KO mice. Percentage of lesion area is the lesion area per total area of aorta. ${ }^{*} P<0.0001$ vs. apoE-KO males; ${ }^{*} P<0.001$ vs. apoE-KO females.

mice. The protein levels of eNOS in apoE-KO/eNOS-Tg mice were also 10.8 times and 3.6 times greater than in WT mice and apoE-KO mice, respectively. Interestingly, in accordance with the report of Laursen et al. (29), each protein level of eNOS in apoE-KO or in apoE-KO/eNOS$\mathrm{Tg}$ mice was slightly, but significantly, increased compared with WT or eNOS-Tg mice, respectively.

$\mathrm{Ca}^{2+}$-dependent NOS activity in aortas was 1.8 times higher in eNOS-Tg mice than in WT mice (eNOS-Tg: $2.73 \pm 0.61 \mathrm{pmol} / \mathrm{mg}$ protein $/ \mathrm{min}$ vs. WT: $1.51 \pm 0.12$ $\mathrm{pmol} / \mathrm{mg}$ protein $/ \mathrm{min} ; P<0.05, n=6$ for each group). ApoE-KO/eNOS-Tg mice also showed a significant increase in NOS activity compared with apoE-KO mice (apoE-KO/eNOS-Tg: $3.05 \pm 0.31 \mathrm{pmol} / \mathrm{mg}$ protein $/ \mathrm{min}$ vs. apoE-KO: $2.10 \pm 0.51 \mathrm{pmol} / \mathrm{mg}$ protein $/ \mathrm{min} ; P<0.05, n=6$ for each group). However, NOS activity in apoE-KO/eNOS-Tg mice did not differ from that in eNOS-Tg mice.

NO production in aortas. The fluorescent signal intensities due to acetylcholine-stimulated NO production were not significantly different between WT and apoE-KO mice (WT mice: $35,680 \pm 8,920 \mathrm{pW} / \mathrm{cm}^{2}$, apoE-KO mice: $32,330 \pm 3,890 \mathrm{pW} / \mathrm{cm}^{2} ; P=\mathrm{NS}$ ). In apoE-KO/eNOS-Tg mice, the endothelium-derived NO production was 1.5 times higher than that in apoE-KO mice (Figure 4, c-g). However, the NO bioactivity in apoE-KO/eNOS-Tg mice was relatively lower than that assumed from the increase in eNOS protein levels.

Increased superoxide production in aortas from apoE$\mathrm{KO} / \mathrm{eNOS}-\mathrm{Tg}$ mice. To investigate the spatial distribution and the quantitative determination of superoxide production in the atherosclerotic vessels, in situ superoxide detection was performed with MCLA. As shown in Figure 5, a and d, the chemiluminescent signal due to superoxide production was hardly detectable in aortas from WT mice. On the other hand, the clear chemiluminescent signal was detected in the atherosclerotic

Table 1

Plasma lipids in apoE-KO mice and apoE-KO/eNOS-Tg mice

\begin{tabular}{|c|c|c|c|c|c|c|}
\hline & & \multirow[t]{2}{*}{ WT (C57BL/6) } & \multicolumn{2}{|c|}{ ApoE-KO } & \multicolumn{2}{|c|}{ ApoE-KO/eNOS-Tg } \\
\hline & & & Male & Female & Male & Female \\
\hline Number & & 7 & 7 & 6 & 8 & 7 \\
\hline $\mathrm{T}-\mathrm{CHO}$ & $\mathrm{mg} / \mathrm{dl}$ & $64.0 \pm 9.0$ & $2059.8 \pm 89.3^{\mathrm{A}}$ & $1959.3 \pm 94.3^{\mathrm{A}}$ & $1841.0 \pm 81.4^{\mathrm{A}}$ & $1981.7 \pm 55.4^{\mathrm{A}}$ \\
\hline HDL-C & $\mathrm{mg} / \mathrm{dl}$ & $31.6 \pm 4.3$ & $66.2 \pm 15.2$ & $43.2 \pm 9.9$ & $61.8 \pm 11.1$ & $45.7 \pm 11.3$ \\
\hline Triglyceride & $\mathrm{mg} / \mathrm{dl}$ & $9.2 \pm 1.2$ & $6.2 \pm 1.5$ & $7.5 \pm 1.0$ & $3.8 \pm 0.8$ & $11.0 \pm 1.7$ \\
\hline
\end{tabular}

ApoE-KO and apoE-KO/eNOS-Tg mice were fed on a high-cholesterol diet for 8 weeks. Animals were fasted for at least 12 hours and bled, and plasma total cholesterol, HDL cholesterol, and triglycerides were determined as described in Methods. ${ }^{A} P<0.01$ vs. WT mice. T-CHO, plasma total cholesterol; HDL-C, HDL cholesterol. 

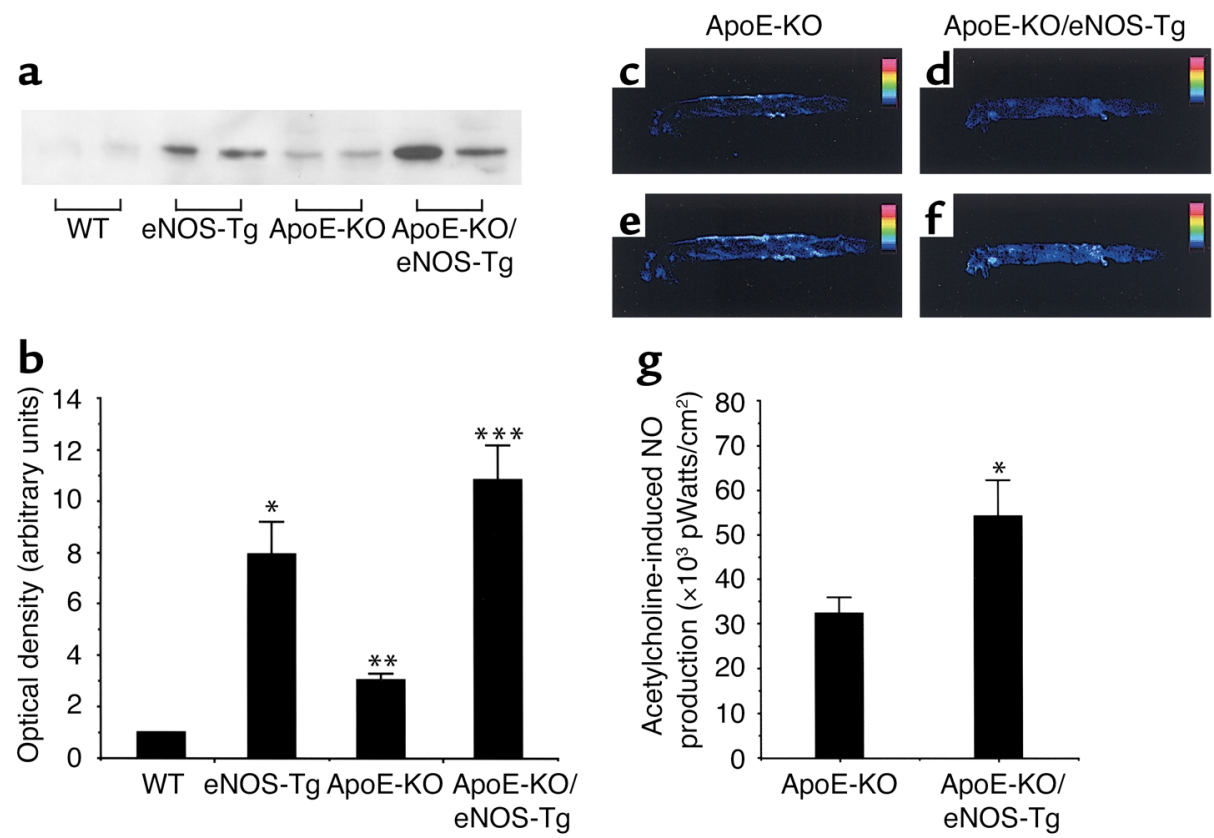

\begin{abstract}
Figure 4
Expression of eNOS and NO production in aortas. (a) Immunoblot analysis for eNOS in aortas. Expression of eNOS in aortas from eNOS-Tg and apoE-KO/eNOS-Tg mice was significantly higher than that in WT mice. Note that the protein levels in aortas from apoE-KO mice or apoE$\mathrm{KO} / \mathrm{eNOS}-\mathrm{Tg}$ mice were not reduced compared with WT mice or eNOS-Tg mice, respectively, but rather increased in the early phase of atherosclerosis. (b) Immunoblots were analyzed and quantified by densitometry. $n=6$ for each group. ${ }^{*} P<0.001$ vs. WT mice; ${ }^{* *} P<0.05$ vs. WT mice; ${ }^{* *} P<0.01$ vs. apoE-KO mice. (c-g) NO production in aortas was evaluated by fluorescence detection with DAF-2 DA as described in Methods. (c-f) Shown are representative photographs of the detected NO production in aortas from apoE-KO (c and e) and apoE-KO/eNOS$\operatorname{Tg}$ mice $(\mathbf{d}$ and $\mathbf{f})$ before $(\mathbf{c}$ and $\mathbf{d})$ and after $(\mathbf{e}$ and $\mathbf{f})$ incubation with acetylcholine $(1 \mu \mathrm{mol} / \mathrm{l})$. NO production was clearly visualized by administration of acetylcholine (e and $\mathbf{f}$ ). (g) Quantitative analysis showed that endothelium-derived NO production in apoE-KO/eNOS-Tg mice was significantly higher than that in apoE-KO mice. Acetylcholine-stimulated NO production was expressed as picowatts per centimeter square. $n=6$ for each group. ${ }^{*} P<0.05 \mathrm{vs}$. apoE-KO mice.
\end{abstract}

plaque lesions of apoE-KO mice (Figure $5, \mathrm{~b}$ and $\mathrm{e}$ ). In apoE-KO/eNOS-Tg mice, MCLA signal was intensely visualized and more extensively distributed from the aortic arch to the iliac bifurcation (Figure 5, $\mathrm{c}$ and $\mathrm{f}$ ).

Quantitative analysis showed that superoxide production in nonplaque areas was increased 3.3-fold in apoE$\mathrm{KO} / \mathrm{eNOS}$-Tg mice and 2.1-fold in apoE-KO mice compared with normal aortic vessels in WT mice (Figure $5 \mathrm{~g}$ ). In plaque areas, superoxide generation was further increased more than tenfold in both apoE-KO and apoE$\mathrm{KO} / \mathrm{eNOS}-\mathrm{Tg}$ mice compared with WT mice. However, the increase in superoxide production was more significantly augmented in both areas of apoE-KO/eNOS-Tg mice compared with those of apoE-KO mice.

To examine the source of superoxide generation in aortas, the endothelium was sequentially removed. In apoE-KO mice, the endothelial denudation resulted in only slight reduction of superoxide production in both plaque and nonplaque areas (Figure $5 \mathrm{~h}$ ). In contrast, superoxide levels in nonplaque areas in apoE$\mathrm{KO} / \mathrm{eNOS}-\mathrm{Tg}$ mice were significantly decreased by the endothelial denudation, showing that the vascular endothelium is the pivotal source of superoxide generation in aortas of apoE-KO/eNOS-Tg mice.

Reduced $\mathrm{BH}_{4}$ contents in atherosclerotic vessels. Enhanced superoxide production and decreased NO bioactivity in the endothelium raised a possibility that activation of eNOS might be dysregulated under hypercholesterolemia in our model. Previous studies demonstrated that loss or insufficiency of $\mathrm{BH}_{4}$, an essential cofactor of NOS, impaired the physiological function of NOS in vitro or in vivo $(18,30,31)$. Therefore, we subsequently measured tissue biopterin contents in aortas to examine the roles of $\mathrm{BH}_{4}$ on the atherogenesis. In apoE- $\mathrm{KO}$ mice, $\mathrm{BH}_{4}$ contents in aortas were approximately 50\% decreased compared with WT mice (WT: $5.13 \pm 0.01 \mathrm{pmol} / \mathrm{mg}$ protein; apoE-KO: $2.56 \pm 0.01$ $\mathrm{pmol} / \mathrm{mg}$ protein; $P<0.01)$. $\mathrm{BH}_{4}$ contents in apoE$\mathrm{KO} / \mathrm{eNOS}-\mathrm{Tg}$ mice were also significantly lower than those in WT mice (apoE-KO/eNOS-Tg: $2.48 \pm 0.01$ $\mathrm{pmol} / \mathrm{mg}$ protein), whereas these $\mathrm{BH}_{4}$ levels in aortas did not differ between apoE-KO and apoE-KO/eNOS$\mathrm{Tg}$ mice. This finding indicates that dysfunctional eNOS was more abundantly present in aortas of apoE$\mathrm{KO} / \mathrm{eNOS}-\mathrm{Tg}$ mice.

Effects of $\mathrm{BH}_{4}$ on the development of atherosclerosis in apoE$\mathrm{KO} / \mathrm{eNOS}$-Tg mice. We hypothesized that supplementation of $\mathrm{BH}_{4}$ could inhibit the progression of atherosclerosis by restoring eNOS function in apoE-KO/eNOS-Tg mice. After 12 weeks of administration, $\mathrm{BH}_{4}$ treatment significantly reduced the lesion size in the aortic tree by $26 \%$ in male and by $28 \%$ in female apoE-KO/eNOS-Tg 

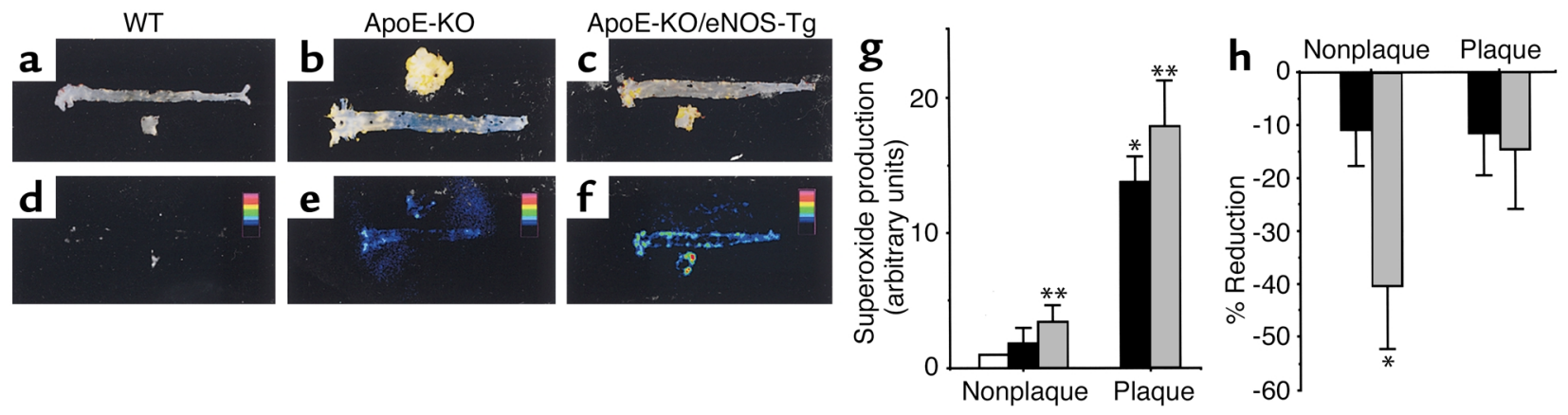

\section{Figure 5}

In situ superoxide detection with MCLA in aortas. Shown are representative photographs for chemiluminescent signal of MCLA (d-f) and each corresponding aorta $(\mathbf{a}-\mathbf{c})$ that was stained with Sudan III from WT mice (a and $\mathbf{d})$, apoE-KO mice (b and $\mathbf{e})$, and apoE-KO/eNOS-Tg mice ( $\mathbf{c}$ and $\mathbf{f}$ ). The chemiluminescent signal due to superoxide production was more widely distributed and more strongly visualized in apoE$\mathrm{KO} / \mathrm{eNOS}-\mathrm{Tg}$ mice compared with apoE-KO mice. (g) Quantitative analysis for superoxide levels in nonplaque and plaque areas in WT mice (white bar), apoE-KO mice (black bars), and apoE-KO/eNOS-Tg mice (gray bars). Superoxide levels were expressed as the ratio (fold increase) to the control values obtained from the normal aortic vessels of WT mice. Superoxide production in apoE-KO/eNOS-Tg mice was significantly increased in both nonplaque and plaque areas compared with apoE-KO mice. $n=6-10$ for each group. ${ }^{*} P<0.05$ vs. normal vessels in WT mice; ${ }^{*} P<0.05$ vs. nonplaque areas in apoE-KO mice; ${ }^{*} * P<0.01$ vs. plaque areas in apoE-KO mice. (h) Reduction of superoxide levels by endothelial denudation in nonplaque and plaque areas of apoE-KO mice (black bars) and apoE-KO/eNOS-Tg mice (gray bars). Shown is the percentage of decreased superoxide production from each predenuded level. In apoE-KO/eNOS-Tg mice, removal of the endothelium resulted in significantly marked decreases in superoxide production in nonplaque areas of the vessels. $n=5-6$ for each group. ${ }^{*} P<0.01$ vs. nonplaque areas in apoE-KO mice.

mice (Figure 6c). In apoE-KO females, the aortic tree lesion size was also decreased by $\mathrm{BH}_{4}$ treatment compared with untreated females (Figure 6d); however, the degree of the reduction size was comparatively smaller than in apoE-KO/eNOS-Tg mice. In contrast, apoE-KO males did not show a significant change in the lesion size under $\mathrm{BH}_{4}$ treatment ( $P=0.25$ vs. untreated males). Plasma lipid profiles were not affected by $\mathrm{BH}_{4}$ administration at 8 or 12 weeks of treatment (data not shown).

Beneficial effects of $\mathrm{BH}_{4}$ on the generation of superoxide and $N O$ in the endothelium. Subsequently, the biochemical effects of $\mathrm{BH}_{4}$ on the production of superoxide and $\mathrm{NO}$ were examined in $\mathrm{BH}_{4}$-treated apoE-KO/eNOS-Tg mice. As shown in Figure 7a, superoxide generation in plaque areas was markedly decreased by $\mathrm{BH}_{4}$ adminis-

\section{Figure 6}

$\mathrm{BH}_{4}$ suppresses atherosclerotic progression in apoE-KO/eNOS-Tg mice. Representative photographs of Sudan III-stained, longitudinally opened aortas from $\mathrm{BH}_{4}$-treated (b) and untreated (a) apoE$\mathrm{KO} / \mathrm{eNOS}-\mathrm{Tg}$ mice on a high-cholesterol diet for 12 weeks. Atherosclerotic lesion formation was remarkably suppressed by $\mathrm{BH}_{4}$ administration in apoE-KO/eNOS-Tg mice. (c) Quantitative analysis of atherosclerotic lesion size in aortas from apoE-KO/eNOS-Tg mice. In $\mathrm{BH}_{4}$-treated apoE-KO/eNOS-Tg mice, atherosclerotic lesion size was significantly smaller than in untreated mice. ${ }^{*} P<0.05$ vs. untreated apoE-KO/eNOS-Tg males; ${ }^{*} P<0.01$ vs. untreated apoE$\mathrm{KO} / \mathrm{eNOS}-\mathrm{Tg}$ females. (d) Quantitative analysis of atherosclerotic lesion size in aortas from apoE-KO mice. $\mathrm{BH}_{4}$ treatment also decreased atherosclerotic lesion size in apoE-KO females; however, the reduction was comparatively smaller than in apoE-KO/eNOS-Tg mice. ApoE-KO males did not show a significant reduction of lesion size. ${ }^{*} P<0.05$ vs. untreated apoE-KO females.

tration (Figure 7a). Superoxide production in nonplaque areas was also slightly decreased by $\mathrm{BH}_{4}$ treatment, although the reduction did not reach statistical significance $(P=0.51)$. Endothelial removal decreased superoxide levels in aortas from either $\mathrm{BH}_{4}$-treated or untreated apoE-KO/eNOS-Tg mice (Figure 7b). However, the degree of the reduction was attenuated by $\mathrm{BH}_{4}$ treatment. These findings show that supplementation of $\mathrm{BH}_{4}$ reduced superoxide production in the endothelium. In addition, acetylcholine-stimulated $\mathrm{NO}$ production was significantly increased by $\mathrm{BH}_{4}$ administration (Figure 7c). Thus, the supplementation of $\mathrm{BH}_{4}$ decreased superoxide generation and increased NO production in the endothelium of apoE-KO/eNOS-Tg mice.

a

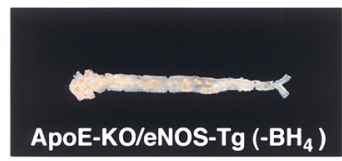

C

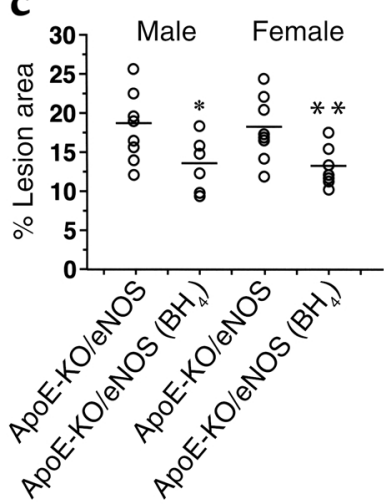

$b$

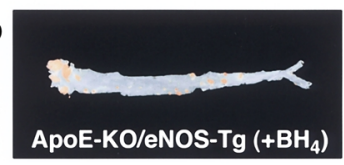

d

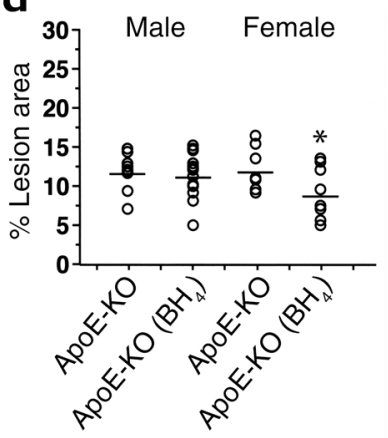


a

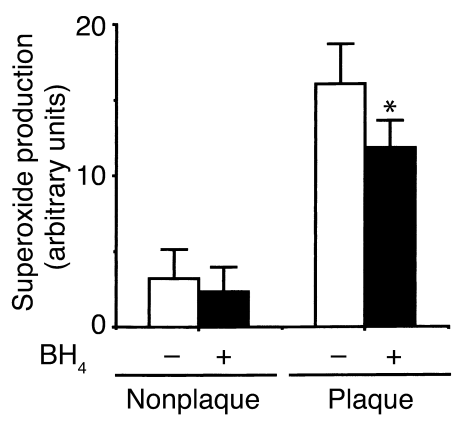

b

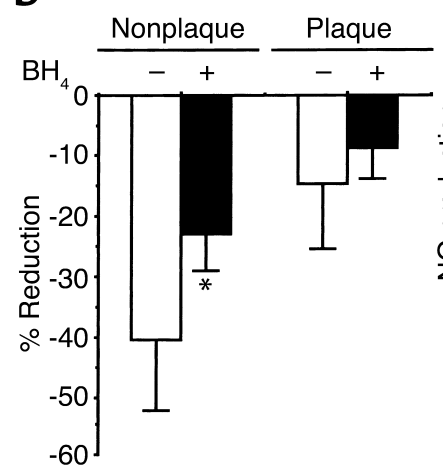

C

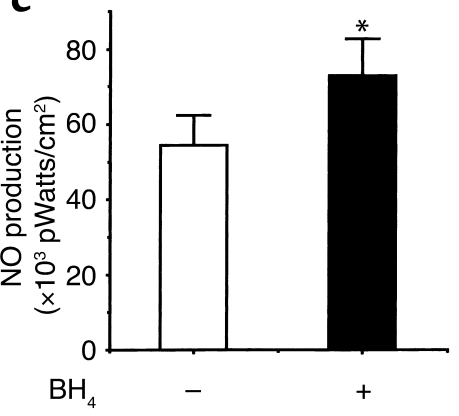

\section{Figure 7}

Reduced superoxide production and increased $\mathrm{NO}$ production in aortas by $\mathrm{BH}_{4}$ supplementation in apoE-KO/eNOS-Tg mice. (a) Quantitative analysis of superoxide production in both nonplaque and plaque areas in $\mathrm{BH}_{4}$-treated (black bars) and untreated apoE- $\mathrm{KO} / \mathrm{eNOS}$ $\mathrm{Tg}$ mice (white bars). $\mathrm{BH}_{4}$ administration significantly decreased superoxide production in plaque areas in apoE-KO/eNOS-Tg. $n=6-10 \mathrm{for}$ each group. ${ }^{*} P<0.01$ vs. plaque areas in untreated apoE-KO/eNOS-Tg mice. (b) Effects of $\mathrm{BH}_{4}$ on the superoxide production in the endothelium. Removal of the endothelium reduced superoxide levels in both nonplaque and plaque areas; however, the degree of the reduction was significantly attenuated in nonplaque areas by $\mathrm{BH}_{4}$ treatment. ${ }^{*} P<0.05$ vs. nonplaque areas in untreated apoE-KO/eNOS-Tg mice. $(\mathbf{c})$ Effects of $\mathrm{BH}_{4}$ on NO production in aortas. Acetylcholine-stimulated $\mathrm{NO}$ production was significantly increased by $\mathrm{BH}_{4}$ supplementation in apoE-KO/eNOS-Tg mice. $n=6$ for both groups. ${ }^{*} P<0.05$ vs. untreated apoE-KO/eNOS-Tg mice.

\section{Discussion}

The roles of eNOS on the development of atherosclerosis have been vigorously investigated. Previous studies reported that eNOS expression was decreased in the severe atherosclerotic lesions in arteries $(32,33)$. Recent studies also showed that eNOS deficiency augmented atherosclerotic lesion formation in apoE-KO mice (34, 35 ). These studies suggest that eNOS acts as an antiatherogenic factor on the vessel walls and that the reduced expression of eNOS precedes more advanced lesion formation of atherosclerosis. Therefore, upregulation of eNOS at the vessel walls has been expected to inhibit the development of atherosclerosis. However, our present study demonstrated that overexpression of eNOS in the endothelium did not inhibit, but accelerated, atherosclerosis in apoE-KO mice.

In the present study, we observed much greater expression of eNOS protein in aortas from apoE$\mathrm{KO} / \mathrm{eNOS}-\mathrm{Tg}$ mice than in apoE-KO mice (Figure 4, a and $b$ ). However, the amount of NO production relative to the eNOS expression level was much lower in apoE-KO/eNOS-Tg mice (Figure 4, $\mathrm{c}$ and d). This finding suggests that there exists dysfunction of overexpressed eNOS, which leads to the decrease in NO bioactivity. In accordance with our present results, it was reported that eNOS expression was maintained or even increased in hypercholesterolemic or atherosclerotic vessels $(29,36,37)$. These studies raise a possibility that the impaired NO bioactivity, rather than eNOS expression, plays an important role in the initiation or progression of atherosclerosis.

Reactive oxygen species generated by a variety of cells existing in the atherosclerotic vessels are profoundly implicated in the pathogenesis of atherosclerosis (38). Superoxide is one of the strongest oxidants and plays a central role as the source of many reactive oxygen species. Therefore, we next examined the superoxide production in aortas of apoE-KO/eNOS-Tg mice. As shown in Figure 5, the superoxide production in apoE$\mathrm{KO} / \mathrm{eNOS}-\mathrm{Tg}$ mice was more extensively distributed and significantly increased not only in the atherosclerotic plaque, but also in nonplaque areas, as compared with apoE-KO mice. Removal of the endothelium significantly decreased superoxide production in nonplaque areas in apoE-KO/eNOS-Tg mice, revealing that the endothelium is a major source of superoxide generation in apoE-KO/eNOS-Tg mice. In contrast, the production of $\mathrm{NO}$ that is capable of scavenging superoxide was also increased in apoE-KO/eNOS-Tg mice (Figure 4). Therefore, the increases in superoxide generation from the endothelium in apoE-KO/eNOS-Tg mice do not seem to result from the reduced production of NO.

In addition to many potential sources of superoxide in the endothelium, such as NADH/NADPH oxidase, xanthine oxidase, and cyclooxygenase (39), NOS has a potency to produce superoxide rather than NO under the conditions in which its substrate L-arginine or cofactors such as $\mathrm{BH}_{4}$ are absent $(10,11)$. It is indicated that lack of or insufficiency of $\mathrm{BH}_{4}$ relative to the eNOS protein amount is responsible for the impaired endothelial function and superoxide production (40, 41). Therefore, as a mechanism of the accelerated atherosclerosis in apoE-KO/eNOS-Tg mice, we explored a possibility that eNOS dysfunction is due to the relative insufficiency of intracellular $\mathrm{BH}_{4}$ levels. Indeed, we found that $\mathrm{BH}_{4}$ contents in aortas were significantly reduced in both apoE-KO and apoE-KO/eNOS-Tg mice compared with those in WT mice. Supplemental $\mathrm{BH}_{4}$ decreased superoxide production in the endothelium of both plaque and nonplaque areas (Figure 7, a and b) and dramatically reduced the size of atherosclerotic lesions in apoE-KO/eNOS-Tg mice (Figure 6c). 
Furthermore, NO production in the endothelium was also significantly increased by $\mathrm{BH}_{4}$ treatment (Figure 7c). Because $\mathrm{BH}_{4}$ is proposed as an antioxidant factor at high doses (42), the reduced atherosclerotic lesion by $\mathrm{BH}_{4}$ treatment might be caused by its antioxidant action. However, the extent of the reduced lesion size was much smaller in $\mathrm{BH}_{4}$-treated apoE-KO mice than $\mathrm{BH}_{4}$-treated apoE-KO/eNOS-Tg mice (Figure 6, $\mathrm{c}$ and d). Therefore, the inhibitory effects of $\mathrm{BH}_{4}$ on the atherosclerotic development were much greater in the presence of eNOS overexpression. This observation suggests that the restoration of eNOS function by $\mathrm{BH}_{4}$ rather than its antioxidant action is mainly responsible for the amelioration of atherosclerotic development. On the other hand, L-arginine is also an essential determinant for NOS activity. It is proposed that its deficiency also causes vascular endothelial dysfunction via a decrease in NO production (43). Although we have not examined the effects of L-arginine treatment on atherogenesis, the possibility can not be excluded that L-arginine also inhibits atherosclerotic progression in apoE-KO/eNOS-Tg mice. Thus, accelerated atherosclerosis by the overexpression of eNOS in apoE-KO mice is at least partly caused by superoxide production from the endothelium through eNOS dysfunction.

Inducible NOS (iNOS) is also shown to be involved in atherogenesis $(21,44,45)$. In immunohistochemistry, we detected iNOS expression mainly in the foam cell-rich regions of atherosclerotic lesions in both apoE-KO and apoE-KO/eNOS-Tg mice. However, the expression of iNOS was not obviously different between the two genotypes (data not shown). In accordance with our findings, Niu et al. reported that the depletion of iNOS did not affect the susceptibility to atherosclerosis in mice (44). In contrast, it was also demonstrated that iNOS accelerated lesion formation from the early phase of atherosclerosis and that tissue injury mediated by iNOS progressed along with lesion formation $(21,45)$. This inconsistency might be due partly to the diversity of atherogenic models; however, the roles of iNOS in atherogenesis might become greater over time with the progression of atherosclerosis. Because the present study was conducted in the relatively early stage of atherosclerosis, the roles of iNOS could be comparatively smaller than the effects of overexpressed eNOS.

Thus, we investigated chronic effects of eNOS overexpression on the development of atherosclerosis using genetically engineered apoE-KO/eNOS-Tg mice. Evidence demonstrates that overexpression of eNOS in the endothelium promoted atherosclerosis in apoE-KO mice. Dysfunction of eNOS that is manifested by the reduced production of $\mathrm{NO}$ and increased generation of superoxide in the endothelium seems to be responsible for the progression of atherosclerosis in apoE$\mathrm{KO} / \mathrm{eNOS}-\mathrm{Tg}$ mice. Furthermore, insufficiency of NOS cofactor $\mathrm{BH}_{4}$ appears to be involved mainly in the eNOS dysfunction. Our study indicates that eNOS function rather than eNOS protein expression is cru- cial for the maintenance or the increase of NO bioactivity. Our present findings also provide insight that modulation of NOS cofactors, including $\mathrm{BH}_{4}$, a major regulator of eNOS, might be a new strategy for the prevention or inhibition of atherosclerosis.

\section{Acknowledgments}

This work was partly supported by the Ministry of Education, Culture, Sports, Science, and Technology of Japan (No. 12470154). The authors thank Shun Ishibashi (Department of Endocrinology and Metabolism, Jichi Medical School) for providing us with apoE$\mathrm{KO}$ mice. We are grateful to Kiyoko Matsui and Ikuyo Kitagawa for experimental assistance.

1. Verbeuren, T.J., et al. 1986. Effect of hypercholesterolemia on vascular reactivity in the rabbit. I. Endothelium-dependent and endothelium-independent contractions and relaxations in isolated arteries of control and hypercholesterolemic rabbits. Circ. Res. 58:552-564.

2. Cooke, J.P., et al. 1992. Antiatherogenic effects of L-arginine in the hypercholesterolemic rabbit. J. Clin. Invest. 90:1168-1172.

3. Cooke, J.P., Andon, N.A., Girerd, X.J., Hirsch, A.T., and Creager, M.A. 1991. Arginine restores cholinergic relaxation of hypercholesterolemic rabbit thoracic aorta. Circulation. 83:1057-1062.

4. Drexler, H., Zeiher, A.M., Meinzer, K., and Just, H. 1991. Correction of endothelial dysfunction in coronary microcirculation of hypercholesterolaemic patients by L-arginine. Lancet. 338:1546-1550.

5. Candipan, R.C., Wang, B.Y., Buitrago, R., Tsao, P.S., and Cooke, J.P. 1996. Regression or progresssion. Dependency on vascular nitric oxide. Arterioscler. Thromb. Vasc. Biol. 16:44-50.

6. Boger, R.H., et al. 1997. Dietary L-arginine reduces the progression of atherosclerosis in cholesterol-fed rabbits: comparison with lovastatin. Circulation. 96:1282-1290.

7. Aji, W., et al. 1997. L-arginine prevents xanthoma development and inhibits atherosclerosis in LDL receptor knockout mice. Circulation. 95:430-437.

8. Channon, K.M., et al. 1998. In vivo gene transfer of nitric oxide synthase enhances vasomotor function in carotid arteries from normal and cholesterol-fed rabbits. Circulation. 98:1905-1911.

9. Qian, H., Neplioueva, V., Shetty, G.A., Channon, K.M., and George, S.E. 1999. Nitric oxide synthase gene therapy rapidly reduces adhesion molecule expression and inflammatory cell infiltration in carotid arteries of cholesterol-fed rabbits. Circulation. 99:2979-2982.

10. Vasquez-vivar, J., et al. 1998. Superoxide generation by endothelial nitric oxide synthase: the influence of cofactors. Proc. Natl. Acad. Sci. USA. 95:9220-9225

11. Xia, Y., Tsai, A.L., Berka, V., and Zweier, J.L. 1998. Superoxide generation from endothelial nitric-oxide synthase. $\mathrm{A} \mathrm{Ca}^{2+} /$ calmodulin-dependent and tetrahydrobiopterin regulatory process. J. Biol. Chem. 273:25804-25808.

12. Ohashi, Y., et al. 1998. Hypotension and reduced nitric oxide-elicited vasorelaxation in transgenic mice overexpressing endothelial nitric oxide synthase. J. Clin. Invest. 102:2061-2071.

13. Ozaki, M., et al. 2001. Reduced hypoxic pulmonary vascular remodeling by nitric oxide from the endothelium. Hypertension. 37:322-327.

14. Kawashima, S., et al. 2001. Endothelial NO synthase overexpression inhibits lesion formation in mouse model of vascular remodeling. Arterioscler. Thromb. Vasc. Biol. 21:201-207.

15. Breslow, J.L. 1996. Mouse models of atherosclerosis. Science. 272:685-688.

16. Piedrahita, J.A., Zhang, S.H., Hagaman, J.R., Oliver, P.M., and Maeda, N. 1992. Generation of mice carrying a mutant apolipoprotein E gene inactivated by gene targeting in embryonic stem cells. Proc. Natl. Acad. Sci. USA. 89:4471-4475.

17. Paigen, B., Morrow, A., Holmes, P.A., Mitchell, D., and Williams, R.A. 1987. Quantitative assessment of atherosclerotic lesions in mice. Atherosclerosis. 68:231-240.

18. Shinozaki, K., et al. 2000. Oral administration of tetrahydrobiopterin prevents endothelial dysfunction and vascular oxidative stress in the aortas of insulin-resistant rats. Circ. Res. 87:566-573.

19. Zhang, S.H., Reddick, R.L., Burkey, B., and Maeda, N. 1994. Diet-induced atherosclerosis in mice heterozygous and homozygous for apolipoprotein E gene disruption. J. Clin. Invest. 94:937-945.

20. Kauser, K., da Cunha, V., Fitch, R., Mallari, C., and Rubanyi, G.M. 2000. Role of endogenous nitric oxide in progression of atherosclerosis in apolipoprotein E-deficient mice. Am. J. Physiol. Heart. Circ. Physiol. 278:H1679-H1685. 
21. Detmers, P.A., et al. 2000. Deficiency in inducible nitric oxide synthase results in reduced atherosclerosis in apolipoprotein E-deficient mice. J. Immunol. 165:3430-3435.

22. Nagata, N., Momose, K., and Ishida, Y. 1999. Inhibitory effects of catecholamines and anti-oxidants on the fluorescence reaction of 4,5diaminofluorescein, DAF-2, a novel indicator of nitric oxide. J. Biochem. (Tokyo). 125:658-661.

23. Itoh, Y., et al. 2000. Determination and bioimaging method for nitric oxide in biological specimens by diaminofluorescein fluorometry. Anal. Biochem. 287:203-209.

24. Qui, W., Kass, D.A., Hu, Q., and Ziegelstein, R.C. 2001. Determinants of shear stress-stimulated endothelial nitric oxide production assessed in real-time by 4,5-diaminofluorescein fluorescence. Biochem. Biophys. Res. Commun. 286:328-335.

25. Yasui, H., and Sakurai, H. 2000. Chemiluminescent detection and imaging of reactive oxygen species in live mouse skin exposed to UVA. Biochem. Biophys. Res. Commun. 269:131-136.

26. Skatchkov, M.P., Sperling, D., Hink, U., Anggard, E., and Munzel, T. 1998. Quantification of superoxide radical formation in intact vascular tissue using a Cypridina luciferin analog as an alternative to lucigenin. Biochem. Biophys. Res. Commun. 248:382-386.

27. Hink, U., et al. 2001. Mechanisms underlying endothelial dysfunction in diabetes mellitus. Circ. Res. 88:E14-E22.

28. Masada, M., Akino, M., Sueoka, T. and Katoh, S. 1985. Dyspropterin, an intermediate formed from dihydroneopterin triphosphate in the biosynthetic pathway of tetrahydrobiopterin. Biochim. Biophys. Acta. 840:235-244.

29. Laursen, J.B., et al. 2001. Endothelial regulation of vasomotion in apoEdeficient mice: implications for interactions between peroxynitrite and tetrahydrobiopterin. Circulation. 103:1282-1288.

30. Huang, A., Vita, J.A., Venema, R.C., and Keaney, J.F. 2000. Ascorbic acid enhances endothelial nitric-oxide synthase activity by increasing intracellular tetrahydrobiopterin. J. Biol. Chem. 275:17339-17406.

31. Heller, R., et al. 2001. L-ascorbic acid potentiates endothelial nitric oxide synthesis via a chemical stabilization of tetrahydrobiopterin. J. Biol. Chem. 276:40-47.

32. Wilcox, J.N., et al. 1997. Expression of multiple isoforms of nitric oxide synthase in normal and atherosclerotic vessels. Arterioscler. Tromb. Vasc. Biol. 17:2479-2488.
33. Oemar, B.S., et al. 1998. Reduced endothelial nitric oxide synthase expression and production in human atherosclerosis. Circulation. 97:2494-2498.

34. Knowles, J.W, et al. 2000. Enhanced atherosclerosis and kidney dysfunction in $\mathrm{eNOS}^{-/-} \mathrm{ApoE}^{-/-}$mice are ameliorated by enalapril treatment. J. Clin. Invest. 105:451-458.

35. Kuhlencordt, P.J., et al. 2001. Accelerated atherosclerosis, aortic aneurysm formation, and ischemic heart disease in apolipoprotein E/endothelial nitric oxide synthase double-knockout mice. Circulation. 104:448-454.

36. Minor, R.L., Myers, P.R., Guerra, R., Bates, J.N., and Harrison, D.G. 1990. Diet-induced atherosclerosis increases the release of nitrogen oxides from rabbit aorta. J. Clin. Invest. 86:2109-2116.

37. Kanazawa, K., et al. 1996. Endothelial constitutive nitric oxide synthase protein and mRNA increased in rabbit atherosclerotic aorta despite impaired endothelium-dependent vascular relaxation. Am. J. Pathol. 148:1949-1956.

38. Dhalla, N.S., Temsah, R.M., and Netticadan, T. 2000. Role of oxidant stress in cardiovascular diseases. J. Hypertens. 18:655-673.

39. Kojda, G., and Harrison, D.G. 1999. Interactions between NO and reactive oxygen species: pathophysiological importance in atherosclerosis, hypertension, diabetes, and heart failure. Cardiovasc. Res. 43:562-571.

40. Cosentino, F., and Luscher, T.F. 1999. Tetrahydrobiopterin and endothelial nitric oxide synthase activity. Cardiovasc. Res. 43:274-278.

41. Katusic, Z.S. 2001. Vascular endothelial dysfunction: does tetrahydrobiopterin play a role? Am. J. Physiol. Heart. Circ. Physiol. 281:H981-H986.

42. Hong, H.J., Hsiao, G., Cheng, T.H., and Yen, M.H. 2001. Supplementation with tetrahydrobiopterin suppresses the development of hypertension in spontaneously hypertensive rats. Hypertension. 38:1044-1048.

43. Kamada, Y., et al. 2001. Vascular endothelial dysfunction resulting from L-arginine deficiency in a patient with lysinuric protein intolerance. J. Clin. Invest. 108:717-724. doi:10.1172/JCI200111260.

44. Niu, X.L., et al. 2001. Inducible nitric oxide synthase deficiency does not affect the susceptibility of mice to atherosclerosis but increases collagen content in lesions. Circulation. 103:1115-1120.

45. Kuhlencordt, P.J., Chen, J., Han, F., Astern, J., and Huang, P.L. 2001. Genetic deficiency of inducible nitric oxide synthase reduces atherosclerosis and lowers plasma lipid peroxides in apolipoprotein E-knockout mice. Circulation. 103:3099-3104. 